\title{
Die Sichtbarmachung einzelner Atome und Moleküle im Feldelektronenmikroskop
}

\author{
Von ERwiN W. MÜLLER \\ Aus dem Kaiser-Wilhelm-Institut für physikalische Chemie und Elektrochemie, Berlin-Dahlem \\ (Z. Naturforschg. 5 a, 473-479 [1950]; eingegangen am 7. August 1950)
}

\begin{abstract}
Die im Feldelektronenmikroskop bei der Adsorption verschiedener Stoffe erscheinende Granulation besteht aus den Streuscheibchen sehr kleiner Teilchen. Eine Reihe von Gründen spricht dafür, daß diese Partikel einzelne Atome oder kleine Moleküle sind, die in einem das gewöhnliche Auflösungsvermögen von etwa $15 \AA$ überschreitenden Abstand hervorstehend adsorbiert sind. Insbesondere stimmt die gemessene Aktivierungswärme der Oberflächenwanderung sowie der Kontrast der Bildpunkte mit den für Einzelatome zu erwartenden Werten überein. Bei einzeln adsorbierten Molekülen konnte durch eine Spreizung der Elektronenbahnen infolge der lokalen Feldverzerrung bei der Erkennbarkeit der Gestalt ein Auflösungsvermögen von $5 \AA$ erreicht werden.
\end{abstract}

$\mathrm{F}$ ür die Sichtbarmachung einzelner Atome in den elektronenoptischen Durchstrahlungsmikroskopen bestehen z.Zt. noch keine günstigen Aussichten. Der kleinste Abstand zweier getrennt wahrnehmbarer Objekte ist immer noch etwa 5-mal größer als die mittleren Atomabstände in den Kristallgittern. Aber auch beim Erreichen des notwendigen Auflösungsvermögens oder mit einer dispersen Anordnung einzelner atomarer Objekte ist der Erfolg wegen des geringen Kontrastes und der Gefahr der Objektbeeinflussung durch die hohe Strahlintensität zweifelhaft ${ }^{1}$. Das elektronenoptische Emissionsmikroskop bleibt wegen zu geringer Auflösung noch weiter zurück, solange die elektrische Feldstärke an der Kathode nicht wesentlich gesteigert werden kann.

Im Gegensatz dazu werden in dem ohne fokussierende Abbildung arbeitenden Feldelektronenmikroskop einzelne Atome unter besonderen Umständen sichtbar. Zwar ist sein Auflösungsvermögen bei einer Abbildung flächenhafter Objekte nur ebenso groß wie das der besten Elektronenmikroskope, aber da für den Kontrast ganz andere Verhältnisse maßgebend sind, können genügend weit auseinanderliegende einzelne Atome als Streuscheibchen in ähnlicher Weise beobachtet werden wie sublichtmikroskopische Objekte im optischen Ultramikroskop.

Das Objekt des Feldelektronenmikroskopes (Abb. 1) ist die kugelige Kalotte einer sehr feinen Metallspitze, die als Kathode im Vakuum einem Leucht-

1 H. B oe r s ch, Z. Physik 127, 391 [1950].

2 E. W. M üll e r, Z. Physik 106, 541 [1937]; 108, 668 [1938]; 126, 642 [1949]. schirm in einigen Zentimetern Abstand gegenübersteht. Dazwischen, aber außerhalb des Strahlenkegels, befindet sich noch eine beispielsweise ringförmige Anode. Beim Anlegen einer Spannung von einigen $1000 \mathrm{~V}$ entsteht an der Spitze eine zur kalten Elektronenemission ausreichende Feldstärke von 3 bis $4 \cdot 10^{7} \mathrm{~V} / \mathrm{cm}$. Die im wesentlichen normal zur Spitzenkalotte austretenden Elektronen ergeben auf dem konzentrischen Leuchtschirm ein Bild der Kathode in einer Vergrößerung, die ungefähr gleich dem Verhältnis von Leuchtschirmradius zu Spitzenradius ist und in der Größenordnung von $10^{5}$ bis $10^{6}$ liegt. Das Bild enthält zunächst die durch ihre unterschiedliche Emission erkennbaren niedrig indizierten Kristallflächen in der Symmetrie, die sich aus dem Anschneiden des Gitters der Spitze durch ihre

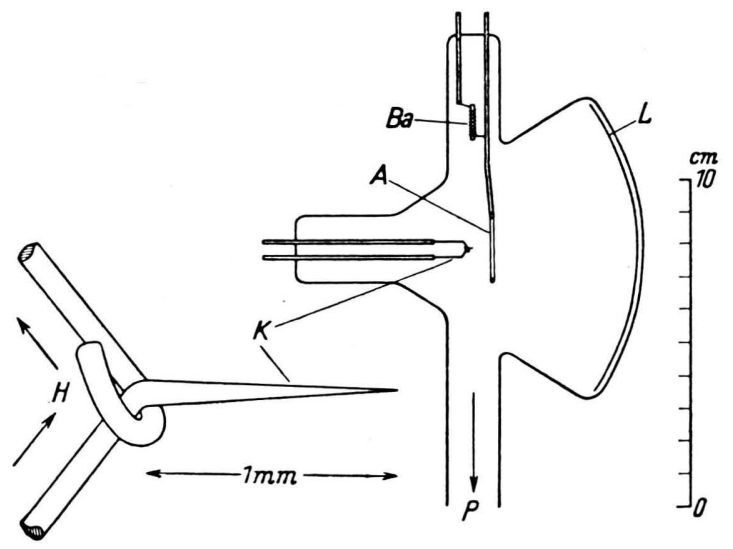

Abb. 1. Feldelektronenmikroskop. H: Heizstrom, K: Kathodenspitze, A: Anodenring, Ba: Barium, L: Leuchtschirm. P: Pumpe. 
Kugelkalotte ergibt. Außerdem zeigt sich aber eine mehr oder weniger deutliche Granulation, die die Unschärfe des Bildes und damit das Auflösungsvermögen ${ }^{3}$ bestimmt. Diese Bildpunkte haben bei den feinsten noch gut zu handhabenden Spitzen einen objektseitigen Durchmesser von 15 bis $20 \AA$. In der zitierten Arbeit konnte gezeigt werden, daß die Körnung auf einem gegebenen Elektronenbild eine einheitliche Größe aufweist und daß man diese Bildpunkte als Streuscheibchen erklären kann, die durch die Tangentialkomponente der Geschwindigkeit der von einem punktförmigen Objekt ausgehenden Elektronen entstehen. In den folgenden $8 \mathrm{Abschnitten}$ sollen verschiedene Gründe dargelegt werden, die es glaubhaft machen, diese punktförmigen Objekte als auf der Oberfläche hervorstehend adsorbierte einzelne Atome anzusehen.

\section{Bildpunkte als Atomanhäufungen}

Die emittierenden Partikel müssen kleiner sein, als unter Berücksichtigung der Vergrößerung objektseitig dem kleinsten erreichbaren Streuscheibchendurchmesser entspricht. Das sind etwa $15 \AA$. Eine Halbkugel von diesem Durchmesser könnte immer noch 55 Wolfram-Atome oder 13 Barium-Atome enthalten und man dürfte also annehmen, daß die Bildpunkte aus zufälligen Agglomeraten von mehreren Atomen bestünden, wobei ihre Helligkeit mit der statistisch gegebenen Streuung der verhältnismäßig geringen Anzahl der Bausteine variiert. Solche Anordnungen von Atomen müßten aber kristallisiert sein, etwa in der von $\mathrm{König}{ }^{4}$ bestimmten Weise in kleinen Elementarwürfeln. Damit sollte aber eine bevorzugte Orientierung dieser Objekte auf gewissen koinzidierenden Bereichen der unterliegenden Kristallkugel ermöglicht werden. Trotz vieler Bemühungen konnten derartige Erscheinungen beim Aufdampfen einer großen Anzahl von Metallen nicht beobachtet werden.

\section{Auflösung eines dispersen Adsorptionsfilmes}

Daß die Bildpunkte einzelne Atome sein könnten, wurde zuerst bei der Beobachtung von BariumFilmen auf Wolfram vermutet ${ }^{5}$. Dampft man Barium auf eine Wolfram-Spitze auf, so ergibt sich nach

3 E. W. M ülle r, Z. Physik 120, 270 [1943].

4 H. K ö ni g, Optik 3, 201 [1948].

5 E. W. M üll e r, Z. Physik 108, 668 [1938].

* Abb 2, s. Tafel S. 480 a. einem Zwischenglühen bei etwa $800^{\circ} \mathrm{K}$ infolge der Oberflächenwanderung ein homogener, „monoatomarer" Adsorptionsfilm mit minimaler Austrittsarbeit, in dem nur die Gebiete um 110, 112 sowie um 100 der Unterlage schwächer emittieren. Die Flächendichte der Barium-Atome ist nicht genau bekannt. D e B o er ${ }^{6}$ rechnet ohne Berücksichtigung der kristallinen Struktur der Unterlage mit $\Theta_{\min }=1,03 \cdot 10^{14}$ Atome $/ \mathrm{cm}^{2}$. Bei dieser dichten Packung beobachtet man im Feldelektronenmikroskop wegen unzureichender Auflösung nur eine homogene Verteilung. Wenn man aber durch schrittweises Abdampfen des BariumFilmes den glühelektrisch meßbaren mittleren Bedeckungsgrad $\Theta$ auf $0,15 \Theta_{\min }$ (bei einer Austrittsarbeit von rd. $4 \mathrm{eV}$ ) erniedrigt, so wird die Adsorptionsschicht körnig (Abb. $2^{*}$ ), weil die mittleren Atomabstände das Auflösungsvermögen überschreiten. Dem Einwande, es könne sich bei dem dispersen Film ebenfalls noch um eine Ansammlung mehrerer Barium-Atome in den einzelnen Objektpunkten handeln, könnte man durch eine Betrachtung der Glühemission derartiger Schichten an Hand der Richardson-Gleichung mit einer ausführlichen Diskussion der Beziehung zwischen Mengenkonstanten und Austrittsarbeit begegnen, worauf aber hier nicht weiter eingegangen sei.

\section{Platzwechselenergie einzelner A tome}

Ein unmittelbarer Nachweis der Sichtbarkeit von einzelnen Atomen ergibt sich aus der Oberflächenwanderung der adsorbierten Partikel ${ }^{7}$. Dampft man auf eine kalte Wolfram-Spitze eine sehr geringe Menge von Wolfram auf, so sieht man insbesondere auf der kaum emittierenden 110-Fläche eine Anzahl sehr schwacher Bildpunkte, die schon bei relativ geringer Erhitzung der Kathode verschwinden. Bei einer Temperatur von $830^{\circ} \mathrm{K}$ dauert es. beispielsweise $200 \mathrm{sec}$, bis diese Teilchen abgewandert sind, bei $1160^{\circ} \mathrm{K}$ sind nur $3 \mathrm{sec}$ dafür erforderlich. Aus solchen Messungen kann man eine Aktivierungsenergie für das Abwandern der Wolfram-Teilchen von etwa $30000 \mathrm{cal} / \mathrm{Mol}$ mit einer Genauigkeit von $\pm 20 \%$ ableiten. Rechnet man in der Weise von Stranski und Suhrmann ${ }^{8}$ aus der Verdamp-

${ }_{6}^{6}$ J. H. d e B o er, Elektronenemission und Adsorptionserscheinungen, Verlag Johann Ambrosius Barth, Leipzig 19.37, S. 73.

7 E. W. M üll e r, Z. Physik 126, 642 [1949].

8 I. N. Stranski u. R. Suhrmann, Ann. Physik 1, 153 [1947]. 
fungswärme des Wolframs die Sattelenergie aus, die beim Platzwechsel eines auf einer intakten 110-Fläche einzeln adsorbierten Wolfram-Atoms aufgebracht werden muß, so ergeben sich hierfür $32600 \mathrm{cal} / \mathrm{Mol}$, also innerhalb der Meßgenauigkeit der experimentell beobachtete Wert. Damit ist die atomare Größe der Partikel nachgewiesen. Wenn es sich um größere Teilchen handelte, so brauchte ihre Auflösung durch Oberflächenwanderung eine viel größere Aktivierungsenergie. Die Abtrennung eines neben einem zweiten Atom adsorbierten Atoms erfordert schon eine Energie von $43800 \mathrm{cal} / \mathrm{Mol}$. Würden vier Atome auf der glatten 110-Fläche des Wolframs beieinander liegen, so wären sogar $56000 \mathrm{cal} / \mathrm{Mol}$ für die Ablösung eines dieser Atome nötig, da die Atome nicht nur von der Unterlage, sondern auch von den mitadsorbierten Nachbarn festgehalten werden.

\section{Platzwechsel auf verschiedenen}

$$
\text { Kristallflächen }
$$

Bei der Betrachtung der Oberflächenwanderung einzelner Partikel sieht man niemals eine seitlich fortschreitende Bewegung, sondern nur das örtlich fluktuierende Aufleuchten und Verschwinden eines Bildpunktes. Das ist auch nicht anders zu erwarten, denn die thermische Translationsgeschwindigkeit einzelner Atome ist viel zu groß. Man kann die Teilchen nur während der Verweilzeit an ihrem Adsorptionsplatz wahrnehmen. Ein disperser Barium-Film erscheint bei Zimmertemperatur vollkommen ruhig, wenn das Vakuum hoch genug ist ( $p \approx 10^{-7}$ Torr) und der Kathodenstrom gering ist (etwa $10^{-6} \mathrm{~A}$ ). Erst beim Erhitzen der Kathode auf etwa $800^{\circ} \mathrm{K}$ beginnen die Bildpunkte zu fluktuieren, indem sie plötzlich erlöschen und an derselben oder einer benachbarten Stelle ebenso schnell wieder erscheinen. Auf einer Wolfram- oder Molybdän-Unterlage beginnt die Oberfächenbeweglichkeit des Bariums bei langsamer Temperatursteigerung zuerst in der weiteren Umgebung von 110 , während die Barium-Atome in den Bereichen um 100 in den sperrigen Vertiefungen, die das Gitter der Unterlage in diesen Flächen bietet, wesentlich fester gebunden sind als auf den Vizinalen der dicht gepackten 110-Flächen. Erst bei etwas höherer Temperatur fluktuieren die Atome auch dort und schließlich wird die Bewegung so heftig, daß nur eine homogene diffuse Fläche erkennbar ist. Im Fall des adsorbierten Cäsiums und Kaliums liegt die-

9 A. Sommerfeld u. H. B ethe, Handbuch d. Physik XXIV/2, S. 441. ser Zustand der Oberflächenbeweglichkeit schon bei Zimmertemperatur vor, man erhält hierbei nicht die körnige Struktur. Dies tritt erst ein, wenn man die Kathode etwa durch Eintauchen der ganzen Röhre in flüssige Luft stark abkühlt.

\section{Berechnung des Kontrastes}

Die theoretische Behandlung der Feldemission eines einzeln auf der Kristallfläche adsorbierten Atoms ist eine sehr schwierige Aufgabe. Man kann den wellenmechanischen Tunneleffekt ${ }^{9}$ nicht mehr wie bei der Emission einer homogenen Oberfläche als ebenes Problem behandeln und man hat vor allem kaum Anhaltspunkte für ein zuverlässiges Modell, das das Elektronensystem des Adatoms mit dem Elektronengas des unterliegenden Metallkristalls gemeinsam darzustellen erlaubt. Um aber überhaupt zu einer Vorstellung von der Größenordnung des von einem Einzelatom zu erwartenden Bildkontrastes zu kommen, wurde im vollen Bewußtsein der Bedenklichkeit des Modells das Adatom als eine leitende Kugel mit derselben Fermi-Kante des obersten Elektronenniveaus und derselben Austrittsarbeit wie die des unterliegenden Metalls betrachtet. Die Feldemission wird bekanntlich aus der Durchlässigkeit der Potentialschwelle an der Metalloberfläche berechnet (Abb. 3). Diese ist

$$
D(w)=e^{-2 \pi \sqrt{8 m} / h \cdot \int_{0}^{x_{1}}(U-w)^{1 / 2} \mathrm{~d} x},
$$

wobei $m$ die Elektronenmasse, $h$ die Plancksche Konstante, $U(x)$ die potentielle und $w$ die kinetische Energie des Elektrons bedeutet. Die Integration ist dabei über die ganze Breite der Potentialschwelle von der Metalloberfläche bis $x_{1}$ zu erstrecken, in der $(U-w)$ positiv ist; unter den vorliegenden Verhältnissen mit $F=4 \cdot 10^{7} \mathrm{~V} / \mathrm{cm}$ und $\Phi=4 \mathrm{eV}$ Austrittsarbeit praktisch etwa von 0 bis $10 \AA$ Abstand von der Oberfläche. Das Potentialprofil $U(x)$ setzt sich dabei

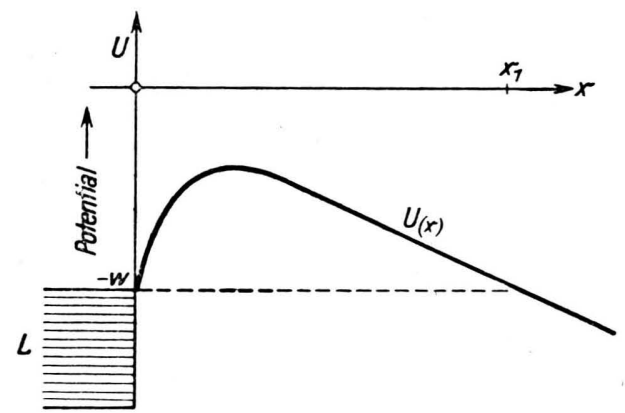

Abb. 3. Potentialverlauf vor der Metalloberfläche. L: Leitungselektronenband. 
aus dem durch das äußere Feld aufgeprägtèn Feldpotential und dem Bildkraftpotential additiv zusammen. Bei unserem Modell mit der leitenden Kugel als Adatom wurde die Potentialverteilung des äußeren Feldes im elektrolytischen Trog ausgemessen. Weiter konnte das Bildkraftpotential durch Messung der elektrostatischen Anziehung einer kleinen, elektrisch aufgeladenen und isoliert aufgehängten Kugel an demselben Oberflächenmodell ermittelt werden. Abb. 4 zeigt das Potentialprofil bei einer glatten Metalloberfläche, das sich aus dem Bildkraftpotential $U=-\frac{1}{4 \pi \varepsilon_{0}} \frac{e}{4 x} ; \quad \varepsilon_{0}=8,86 \cdot 10^{-14} \frac{\mathrm{Coul}}{\mathrm{V} \mathrm{cm}}$ und dem linearen Potentialabfall $U=E x$ (bei $E=4 \cdot 10^{7} \mathrm{~V} / \mathrm{cm}$ äußerer Feldstärke zusammensetzt.) Für ganz kleine Abstände unter $1,5 \AA$ wird schließlich nach Schott $\mathrm{ky}^{10}$ ein linearer Potentialabfall bis zur Metalloberfläche angenommen. Außerdem ist das am Modell gemessene Bildkraftpotential und das Raumpotential für eine aufsitzende Kugel von 4,35 $\AA$ Durchmesser (Barium-Atom) dargestellt. Im Falle des Adatoms beginnt dabei die $x$-Koordinate nicht an der Metallunterlage, sondern an dem hervorstehenden Pol der Kugel. Durch Summation erhält man jedesmal das Profil des Potentialberges, den die Elektronen mit Hilfe des Tunneleffektes durchdringen müssen. Die Ermittlung der Durchlässigkeit erfolgt durch graphische Integration nach dem Radizieren. Setzt man die Durchlässigkeit der glatten Fläche $D=1$, so ist die über einer adsorbierten aufsitzenden Kugel von $2,75 \AA$ Durchmesser (Wolfram-Atom, in Abb. 4 nicht

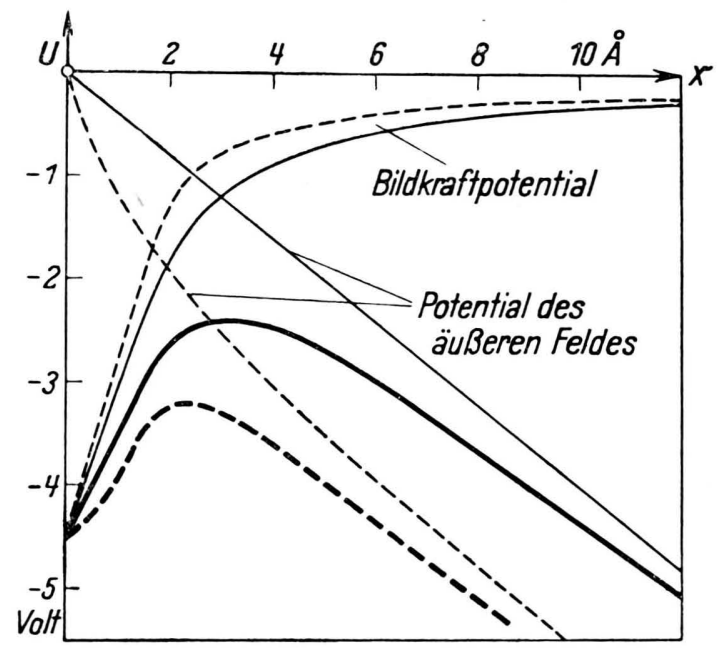

Abb. 4. Potentialwall bei einer äußeren Feldstärke von $4 \cdot 10^{7} \mathrm{~V} / \mathrm{cm}$ vor einer Ebene (ausgezogen) und vor einer einzeln auf ihr sitzenden Kugel von 4,3 $\AA$ Durchmesser (Ba-Atom) (gestrichelt). dargestellt) $D_{\mathrm{W}}=81$, die über einer Kugel von $4,35 \AA$ A Durchmesser (Barium-Atom) $D_{\mathrm{Ba}}=650$. Die sehr viel größere Durchlässigkeit der Potentialschwelle im Falle des Barium-Atoms im Vergleich zum adsorbierten Wolfram-Atom ergibt sich daraus, daß nicht die Feldstärke unmittelbar an der Kugeloberfläche selbst, sondern das Profil über den ganzen Potentialberg maßgebend ist, wodurch das große Barium-Atom sehr viel wirksamer wird. Setzt man in unserem einfachen Modell die Elektronenemission proportional der Durchlässigkeit bei $w=4,5 \mathrm{eV}$, und sieht man diese außerdem über einer Fläche von dem Durchmesser des Atoms $\delta$ als homogen an, so kann man sogleich die in den Bildpunkten vom objektseitigen Durchmesser $d$ entstehende relative Stromdichte angeben, da diese proportional zu $D(\delta / d)^{2}$ ist. Beim Streukreisdurchmesser von $20 \AA$ verhalten sich damit die Stromdichten im Bildpunkte einer glatten Kristallfläche bzw. eines aufsitzenden einzelnen Wolframund Barium-Atoms etwa wie $1: 2,5: 31$.

Diese berechneten Kontraste stimmen mit der Beobachtung in der Größenordnung durchaus überein. Sie sind in Wirklichkeit etwas geringer, was nicht nur auf die Unvollkommenheit des Modells zurückzuführen ist. Die Einzelatome werden vorzugsweise gar nicht auf glatten Kristallfächen adsorbiert sein, sondern sich bei ausreichender Oberflächenbeweglichkeit an den Netzebenenstufen und Wachstumsstellen anlagern, so daß ihre lokale Feldstärkenerhöhung durch eine partielle Abschirmung vermindert wird. Insbesondere werden sich Wolfram-Atome oder andere gleich große oder gar kleinere Atome dann der Beobachtung entziehen, während die großen BariumAtome an solchen Adsorptionsstellen immer noch weit genug hervorragen, um eine erhöhte Feldemission zu geben, die aber entsprechend der Abschirmung örtlich sehr verschieden sein kann. So liegen die BariumAtome in den sperrigen Vertiefungen auf den 100und 111-Flächen bedeutend tiefer adsorbiert als auf den dicht gepackten 110-Flächen des Wolframs. Daher erscheinen die Barium-Punkte in Abb. 2 auf den erstgenannten Flächen auch viel schwächer als auf den Vizinalen der 110-Fläche. Auf dieser Fläche selbst wird Barium nicht adsorbiert. Es wäre leicht, an Hand weiterer Oberflächenmodelle die Feldverhältnisse auszumessen, wenn das Adatom die genannten ausgezeichneten Lagen auf den verschiedenen Kristallflächen einnimmt, um aus der wirksamen lokalen Feldstärke nähere Bedingungen für die Elektronen-

10 W. S chottky, Z. Physik 14, 63 [1923]. 
emission und damit die Kontrastverhältnisse abzuleiten. Bei der Unvollkommenheit aber, mit der der wirkliche Emissionsmechanismus von solchen Modellen her erfaßt werden kann, dürfte es besser sein, die Sichtbarkeitsbedingungen für Einzelatome nicht weiter als im folgenden zu präzisieren: Die Teilchen müssen in einem das Auflösungsvermögen überschreitenden Abstande auf glatten Kristallflächen möglichst weit hervorstehend adsorbiert sein und ihr Durchmesser soll möglichst groß sein, jedenfalls nicht kleiner als der der Unterlageatome. Im letzten Grenzfalle sind Einzelatome eben noch zu erkennen, wenn sie auf dichtest besetzten vollständigen Netzebenen der Unterlage aufsitzen.

Nachdem die Frage des Bildkontrastes eindeutig für die Sichtbarkeit einzelner Atome entschieden ist, bleibt noch zu bedenken, ob durch die ungeheure Strombelastung nicht eine Objektzerstörung durch Verdampfen des Atoms eintreten muß. Ein Bildpunkt hält im Falle des Bariums eine Stromstärke von $10^{-7}$ A gerade noch aus. Dem entspricht eine Stromdichte von fast $10^{5} \mathrm{~A} / \mathrm{cm}^{2}$. Etwa ${ }^{1 / 10}$ aller vom Metallinnern gegen die Oberfläche anlaufenden Elektronen in einem Bereich von $0,5 \mathrm{~V}$ Breite unterhalb der Fermi-Kante tritt hier aus. Bei dieser Beobachtung beträgt die Gesamtstromstärke der Kathode etwa $10^{-4}$ A. Eine Spitze von $10^{-5} \mathrm{~cm}$ Radius wird dabei durch Joulesche Wärme auf schätzungsweise $800^{\circ} \mathrm{K}$ aufgeheizt.

\section{Kathodenzerstäubung}

Bei niedrigen Kathodentemperaturen und nicht allzu großen Stromstärken erfolgt das spontane Verschwinden der Adatome offenbar durch die Kathodenzerstäubung infolge der einfallenden Ionen. Die von dem Einzelatom ausgehenden Elektronen erreichen in etwa $40 \AA$ Entfernung die zur Ionisation des Restgases notwendige Geschwindigkeit. Mit der in zunehmender Entfernung von der Kathode größer werdenden Geschwindigkeit nimmt auch die Ionisationswahrscheinlichkeit beim gaskinetischen Zusammenstoß $\mathrm{zu}$, doch trifft nur ein kleiner Teil der so erzeugten Ionen beim Herunterfallen auf die Kathode das Adatom selbst, da sich der Elektronenstrahl schon in $40 \AA$ Abstand auf einen Durchmesser von $20 \AA$ verbreitert hat. Durch genauere Betrachtung der Elektronenbahnen läßt sich die Zahl der das Adatom treffenden Ionen berechnen. Sie ist proportional dem aus der Kathode kommenden Elektronenstrom, dem Gasdruck und dem graphisch auszuwertenden Weg- integral der Ionisierungsfunktion. Nimmt man als Restgas Stickstoff an (die Ionisierungsfunktion ist auch für andere Gase nicht größenordnungsmäßig verschieden), so erhält man bei einem Vakuum von $10^{-6}$ Torr und einem vom Einzelatom ausgehenden Strom von $10^{-8} \mathrm{~A}$ größenordnungsmäßig alle $10 \mathrm{sec}$ einen Ionentreffer auf dem Adatom. Die einfallenden Ionen haben alle möglichen Energien, von $10 \mathrm{eV}$ bis $\mathrm{zu}$ einigen $1000 \mathrm{eV}$, jedoch ist die Treffwahrscheinlichkeit für die in großer Entfernung von der Spitze entstandenen schnellen Ionen sehr gering. Dennoch dürfte jeder Treffer das Adatom entweder verdampfen oder zumindest von dem innegehabten Platz wegstoßen. Tatsächlich ist die Geschwindigkeit des Fluktuierens unter den genannten Bedingungen auch durch eine mittlere Lebensdauer der Bildpunkte von etwa $10 \mathrm{sec}$ zu beschreiben. Wegen der Druckproportionalität der Kathodenzerstäubung kann man aus der Häufigkeit des Fluktuierens den in der Röhre herrschenden Gasdruck der Größenordnung nach abschätzen. Bei einzelnen sehr hellen Bildpunkten, die zweifelsohne Agglomerate aus mehreren Atomen sind, bemerkt man gelegentlich eine stufenweise Intensitätsänderung, die offenbar durch den sukzessiven $\mathrm{Ab}$ bau einzelner Teilchen hervorgerufen wird. Auch aus den Beobachtungen der Kathodenzerstäubung kann man also zwanglos die Sichtbarkeit atomarer Einzelobjekte bestätigen.

\section{Sichtbarkeit einzelner Moleküle}

Die wichtigste Bedingung für die Sichtbarkeit von Teilchen mit geringerer Größe als dem Auflösungsvermögen entspricht, nämlich die zur lokalen Feldstärkeerhöhung erforderliche hervorstehende Anlagerung, ist für größere Moleküle natürlich leichter zu erfüllen als für einzelne Atome. Man erhält daher auch beim Aufbringer verschiedener molekularer Stoffe die für feine Partikel charakteristische Granulation sowie in einigen Fällen spezifische Orientierungseffekte auf der kristallinen Unterlage von geradezu analytischer Verwendbarkeit.

Durch die Eigenart der Elektronenbahnen unmittelbar vor dem Objekt kann besonders bei flachen Molekülen auch eine Abbildung der Gestalt von Teilchen zustandekommen, deren Abmessungen noch weit unterhalb $10 \AA$ liegen und damit kleiner sind als dem Auflösungsvermögen eigentlich entspricht. Dampft man z. B. Phthalocyanin oder Hämin (Abb. 5) auf die Wolfram-Spitze auf, so erhält man in Abb. $6^{*}$,

* Abb. 6, 7 u. 8 s. Tafel S. 480 b. 


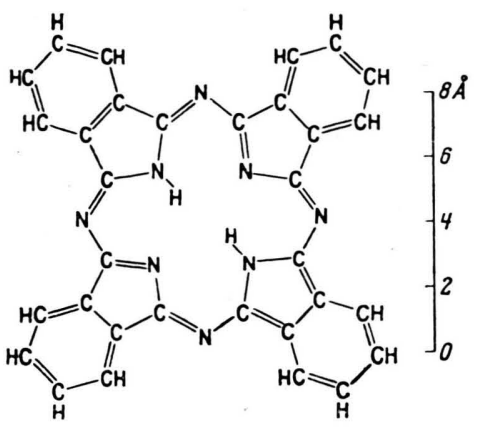

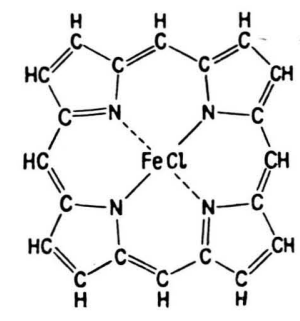

Abb. 5. Phthalocyanin-Molekül und Porphyrin-Ring des Hämins.

7 und 8 deutlich die Symmetrie des aus der Röntgenfourieranalyse bekannten Moleküls ${ }^{11}$. Die Mitten der vier Benzolringe an den Ecken des Phthalocyaninmoleküls haben 7,7 $\AA$ Abstand voneinander, beim Porphyrin-Ring des Hämins werden auch die PyrrolRinge mit nur $5 \AA$ Abstand getrennt. Es muß sich hier um Bilder von einzelnen Molekülen handeln, denn eine quadratischeZusammenlagerung von genau 4 Molekülen beim Aufdampfen ist ganz unwahrscheinlich. Außerdem sieht man bei der Betrachtung auf dem Leuchtschirm ziemlich oft, wie sich die Moleküle um ihren Mittelpunkt drehen, bis sie zur Wolfram-Unterlage eine bestimmte Richtung eingenommen haben. Diese Moleküle sind wegen ihrer großen Einfangfläche gegen einfallende Ionen außerordentlich empfindlich, und da das Vakuum beim Aufdestillieren kaum besser als $10^{-6}$ Torr sein kann, beträgt die Verweilzeit bei größeren zur Leuchtschirmphotographie ausreichenden Bildhelligkeiten nur wenige Sekunden.

Im einzelnen beobachtet man beim Aufdampfen derartiger Moleküle folgendes:

a) Auf einer sehr reinen Wolfram-Unterlage erscheinen die aus hoch entgastem Phthalocyanin aufgedampften Moleküle zunächst als feine Punkte ganz wie die Bilder größerer Einzelatome oder gewöhnlicher kleiner Moleküle.

b) Mit zunehmender Aufdampfmenge, die aber immer noch geringer ist als etwa monomolekularer Bedeckung entspricht, erscheinen häufig Doppelbildpunkte und schließlich auch vierteilige Molekülbilder.

c) Bei noch größerer Aufdampfmenge sind einige Molekülbilder bis etwa 8-mal größer, als sich aus der linearen Vergrößerung des Mikroskops ergibt.

d) Man kann auch gleich bei geringster Aufdamp615.

11 J. M. Ro berts o n, J. chem. Soc. [London] 1935, fung "große" vierteilige Molekülbilder erhalten, wenn die Wolfram-Unterlage vorher mit geeigneten Fremdatomen oder Molekülen bedeckt war. Solche Fremdstoffe können auch aus unvollkommen entgastem Phthalocyanin selbst kommen.

e) Das etwas kleinere Hämin-Molekül, das bei dem Aufdestillieren bei $360^{\circ} \mathrm{C}$ wahrscheinlich nur noch aus dem Porphyrin-Gerüst besteht, ist sehr viel seltener zu sehen. Doppelbildpunkte sind verhältnismäßig häufig, aber vierteilige Molekülbilder können im ganzen Bildfeld gleichzeitig nur ein- bis dreimal gefunden werden.

Diese Beobachtungen lassen sich bei Betrachtung der lokalen Feldverhältnisse vor den adsorbierten Molekülen zwanglos deuten. In Abb. 9 ist im Schnitt ein Adatom, ein unmittelbar auf der Unterlage adsorbiertes flaches Molekül und ein auf anderen adsorbierten Teilchen aufliegendes Molekül mit den durch die lokale Verzerrung des äußeren Feldes geformten Elektronenbahnen unter der Annahme dargestellt, daß die aufliegenden Teilchen ebenfalls „Kathodenpotential“ besitzen. Das Elektronenbüschel des Adatoms erzeugt auf dem Leuchtschirm den Streukreis, der, auf die Objektebene zurückprojiziert, etwa $20 \AA$ Durchmesser hat. Von den infolge lokaler Feldstärkeerhöhung stark emittierenden Ecken der adsorbierten Moleküle verlaufen die Elektronenbahnen nach außen durchgebogen, wodurch ihre Streukreise so weit auseinander zu liegen kommen, $\mathrm{da} ß$ sie in der Objektebene einen viel größeren $\mathrm{Ab}$ stand zu haben scheinen, als den Abständen der Molekülecken entspricht. Diese „Abbildungsspreizung“ hängt in ihrem Ausmaße stark von der Höhe ab, in der das Molekül über der in erster Näherung als eben anzusehenden Unterlage angelagert ist. Wie sich aus

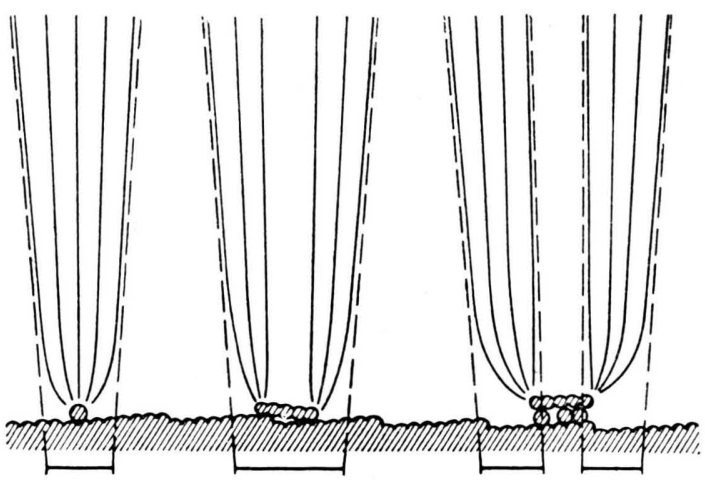

Abb. 9. Streukreise eines Einzelatoms, eines aufliegenden und eines auf zwischenliegenden Atomen adsorbierten flachen Moleküls mit den gespreizten Elektronenbahnen. 
Abb. 9 ergibt, ist eine wesentliche Spreizung erst bei einer Adsorption über zwischenliegenden Fremdatomen bzw. kleinen Molekülen zu erwarten. Damit sind auch die beim Aufdampfen gewonnenen Erfahrungen zu verstehen. Die zuerst erscheinenden Doppelbildpunkte rühren von den zwei hervorstehenden Molekülecken her, wenn sich das Molekül schräg auf einzelne Unterlageatome stützt. Erst bei größerer Fremdatombedeckung kommt es häufiger vor, daß das Molekül drei oder mehr Unterlageteilchen findet und dann flach adsorbiert wird. Die nähere Umgebung dieser günstigen Adsorptionsstelle muß natürlich frei von anderen Fremdatomen sein, damit das Molekül genügend weit hervorsteht. Bei dem recht kleinen Hämin-Molekül wird die Wahrscheinlichkeit für das Eintreffen derartig günstiger Unterlagekombinationen sehr gering sein, von der Größenordnung $10^{-3}$, so daß man diese Moleküle so selten flach adsorbiert vierteilig findet.

\section{Objekttreue der Abbildung}

An der Grenze des Auflösungsvermögens ist die Objektähnlichkeit einer Abbildung schon definitionsgemäß fragwürdig. Die Ähnlichkeit der durch die Strahlspreizung vermittelten Abbildung unterliegt natürlich noch weiteren Einschränkungen. So sieht z. B. das Cu-Phthalocyanin, das in der Mitte statt der zwei Wasserstoffatome ein Kupferatom enthält, genau so aus wie das metallfreie Molekül ${ }^{12}$. Das Zentralatom ist trotz seiner Größe so weit abgeschirmt, daß sein mitten im Gesamtbild erscheinender Streukreis, verglichen mit der starken Emission der vier

12 E. W. M ülle r, Naturwiss. 37, 333 [1950].
Ecken, intensitätsmäßig stark zurückbleibt. Auch der in seinem Aufbau ebenfalls quadratische PorphyrinRing gibt nach Abb. 7 und 8 ein dem Phthalocyanin sehr ähnliches Bild, jedoch ist die mehr geschlossene Form des Moleküls deutlich zu erkennen. Es gibt beim Hämin zwei verschiedene Arten von Bildern mit Vierersymmetrie, eine ist, ähnlich dem Phthalocyanin, in der Mitte dunkel (Abb. 7), die andere in Abb. 8 wiedergegebene zeigt im Mittelpunkt noch eine Aufhellung. Man geht wohl nicht fehl in der Annahme, hier das am zentralen Eisen-Atom gebundene Chlor-Ion unmittelbar zu sehen, das auf einer Seite des flachen Moleküls hervorsteht und nur dann sichtbar wird, wenn diese Seite dem Leuchtschirm zugewandt ist.

Mehr als den Nachweis der quadratischen Form dieser kleinen Moleküle darf man mit dem erreichten Auflösungsvermögen von $5 \AA$ und der zusätzlichen Beschränkung der Abbildung durch die lokalen Feldverhältnisse nicht erwarten. Versuche mit ähnlichen flachen, aber unsymmetrischen Molekülen, z. B. mit Chlor teilweise substituierten Phthalocyaninen sowie in mehr als atomarer Schichtdicke aufgedampften Metallen, die dann unter Umständen submikroskopische Kriställchen von stark variierender Größe zu bilden scheinen, sind im Gange. Dabei wird eine wesentliche Aufgabe die Analyse der gespreizten Elektronenbahnen unmittelbar vor dem Objekt sein, um damit aus den erhaltenen Bildern auf seine wahre Gestalt schließen zu können.

Zum Schluß möchte ich auch hier Hrn. Prof. Dr. I. N. $\mathrm{S}$ transki für sein durch stetige Förderung gezeigtes Interesse herzlich danken. 